GNPHE/10-03

\title{
Engineering of Quantum Hall Effect from Type IIA String Theory on The K3 Surface
}

\author{
Adil Belhaj ${ }^{1,3 *}$, Antonio Segui ${ }^{2 \dagger}$ \\ ${ }^{1}$ Centre National de l'Energie, des Sciences et des Techniques Nucleaires, CNESTEN \\ Rabat, Morocco \\ ${ }^{2}$ Departamento de Física Teórica, Universidad de Zaragoza, E-50009-Zaragoza, Spain \\ ${ }^{3}$ Groupement National de Physique des Hautes Energies, GNPHE, Siège focal: FSR \\ Rabat, Morocco
}

August 21, 2018

\begin{abstract}
Using D-brane configurations on the K3 surface, we give six dimensional type IIA stringy realizations of the Quantum Hall Effect (QHE) in 1+2 dimensions. Based on the vertical and horizontal lines of the K3 Hodge diamond, we engineer two different stringy realizations. The vertical line presents a realization in terms of D2 and D6-branes wrapping the K3 surface. The horizontal one is associated with hierarchical stringy descriptions obtained from a quiver gauge theory living on a stack of D4-branes wrapping intersecting 2-spheres embedded in the K3 surface with deformed singularities. These geometries are classified by three kinds of the Kac-Moody algebras: ordinary, i.e finite dimensional, affine and indefinite. We find that no stringy QHE in $1+2$ dimensions can occur in the quiver gauge theory living on intersecting 2-spheres arranged as affine Dynkin diagrams. Stringy realizations of QHE can be done only for the finite and indefinite geometries. In particular, the finite Lie algebras give models with fractional filling fractions, while the indefinite ones classify models with negative filling fractions which can be associated with the physics of holes in the graphene.
\end{abstract} Keywords: Quantum Hall Effect, Type IIA superstring, K3 surface, Quiver gauge models.

*belhaj@unizar.es

${ }^{\dagger}$ segui@unizar.es 


\section{Introduction}

Susskind has conjectured that a two-dimensional quantum Hall fluid of charged particles with

filling fraction $\nu=\frac{1}{k}$ is described by a non-commutative Chern-Simons gauge theory at level $k$ [1]. This conjecture has opened a new way to apply string theory to study low-energy systems in condensed matter physics as the Quantum Hall Effect (QHE) in $1+2$ dimensions. This has been based on the recent developments in string dualities and brane physics. Roughly, the first connection with string theory has been given by Bernevig, Brodie, Susskind and Toumbas to reproduce the QHE on the 2-sphere, $S^{2}$ [2]. The corresponding brane configuration involves a spherical D2-brane and dissolves D0-branes on it. The system has been placed in a background of coincident D6-branes extended in the directions perpendicular to the world-volume of the D2-brane on which QHE resides. Some further details on this construction can be found in [3]-[10]. However, this type IIA superstring construction has been done in ten dimensional space-time. Therefore a natural question arises whether the QHE in $1+2$ dimensions can be realized in terms of lower dimensional type IIA superstring spectrums.

The aim of this work is to address this question by giving stringy realizations of the QHE in $1+2$ dimensions from a six dimensional string space-time point of view. Motivated by the paper [11] and the results obtained on the attractor horizon geometries of the extremal black 2-branes [12, 13, we engineer brane representations of the QHE in 1+2 embedded in the compactification of type IIA superstring on the K3 surface. Based on the vertical and horizontal lines of the Hodge diamond of the K3 surface, we give two different stringy realizations. The vertical line is associated with a realization in terms of D2 and D6-branes wrapping the K3 surface. However, the horizontal line corresponds to a quiver gauge theory realization of the QHE in 1+2 dimensions. This gives hierarchical stringy descriptions in terms of wrapped D4-branes on intersecting 2-spheres of local limits of the K3 surface. The intersection numbers are encoded in the Cartan matrices classified by three kinds of the Kac-Moody algebras [14, 15, 16]. They are known as: ordinary i.e finite dimensional, affine and indefinite. We find that no stringy QHE in 1+2 dimensions can occur in the quiver gauge theory living on a stack of D4-branes based on the affine Dynkin diagrams. However, the finite Lie algebras classify models with fractional filling fractions; while the indefinite ones give models with negative filling fractions, which could be associated with the holes in the graphene physics.

The organization of this paper is as follows. In section 2 we review briefly the stringy realization of the QHE in $1+2$ dimensions in terms of ten dimensional type IIA superstring. In section 3, we give a six dimensional stringy realizations of the QHE in $1+2$ dimensions from the compactification of type IIA superstring on the K3 surface. Based on the Hodge diamond vertical line of the K3 surface, we give a realization in terms of D2 and D6-branes wrapping the K3 surface. In section 4, we present hierarchical stringy descriptions obtained from quiver 
gauge theories living on the world-volume of D4-branes wrapping intersecting 2-spheres of local limits of the K3 surfac with geometries classified bt the three kinds of Kac-Moody Lie algebras. These realizations, which are based on the horizontal line, lead to the QHE with filling fractions depending on local type IIA geometries. The last section is devoted to discussions and open questions.

\section{Uncompactified String Theory Realization of the QHE}

Following [2], 3-dimensional QHE systems of condensed matter physics can be modeled by low energy dynamics of non perturbative extended objects, involving D0, D2 and D6-branes of uncompactified type IIA superstring living in ten dimensions. To see that, consider first a D2-brane, with a spherical configuration, and dissolves $N$ D0-branes moving on it. Second we take a stack of D6-branes with $K$ charges placed in the directions perpendicular to the ones where the spherical D2-brane lives. Then we move them to the center of the 2-sphere. In this brane configuration, the world-volume of the D2-brane plays the role of the space-time of the 3-dimensional QHE systems. It can be also interpreted as the space-time of the 3-dimensional Chern Simons theory with $U(1)$ gauge group living on the D2-brane. The D6-branes, located at the origin of the D2-brane, can be thought of as an external source of the magnetic charges. This has been required from the fact that in ten dimension a $p$-dimensional electrical brane is dual to a $q$-dimensional magnetic one such that

$$
p+q=6 .
$$

When the D6-branes cross the D2-brane, the Hanany-Witten effect produces fundamental strings which are stretched between D2 and D6-branes [17]. In this way, the strings ending on the D2-brane have an interpretation in terms of the fractional quantum Hall particles (Hall electrons). The D0-branes behave as a magnetic flux in the world-volume of the D2-brane and the string ends are charged under the U(1) world-volume gauge field. The number of the D6-branes $K$ can be identified with the number of charged particles and $N$ is the total magnetic flux. For this brane configuration, the ratio

$$
\nu=\frac{K}{N}
$$

determines the filling fraction of the system.

At this level, one might naturally ask the following question. Is there any compactified string theory realization of the QHE in $1+2$ dimensions?. In what follows, we address this question using the compactification of type IIA superstring on the K3 surface and wrapped D-branes on appropriate cycles. 


\section{Six Dimensional Type IIA String Realization of the QHE}

Using the results of [11] and the black brane attractors on the K3 surface [12, 13], we engineer six dimensional string realizations of the QHE in $1+2$ dimensions. This can be embedded in the compactification of type IIA superstring on the K3 surface. This geometry is a two dimensional complex Calabi-Yau manifold admitting a $S U(2)$ holonomy group. It has many types of realizations; the simplest one is to consider the orbifold $T^{4} / G$ where $G$ is a subgroup of $S U(2)$. In the case of $G=Z_{2}$, the $\mathrm{K} 3$ surface is obtained by blowing up the sixteen singular fixed points of the corresponding orbifold. Each fixed point is replaced by a 2 -sphere $S^{2}$. The K3 surface involves a Hodge diamond playing a crucial role in the determination of the six dimensional string theory spectrum and the corresponding brane configurations. For a generic K3 surface, the Hodge diamond is given by

$$
\begin{aligned}
& h^{0,0}=1 \\
& h^{1,0}=0 \quad h^{0,1}=0 \\
& h^{2,0}=1 \quad h^{1,1}=20 \quad h^{0,2}=1 . \\
& h^{2,1}=0 \quad h^{1,2}=0 \\
& h^{2,2}=1
\end{aligned}
$$

This graph shows that $H_{2}(K 3)$ has dimension 22, corresponding to the fact that $h^{2,0}=h^{0,2}=1$ and $h^{1,1}=20$. The compactification of type IIA superstring on the K3 surface is obtained by breaking the space-time symmetry $S O(1,9)$ down to the subgroup $S O(1,5) \times S U(2)$ which is contained in $S O(1,5) \times S O(4)$. More details on this compactification can be found in [18].

Similarly as for the ten dimensional realization of the QHE in $1+2$ dimensions, the spacetime is described by the world-volume of membranes embedded in the resulting six dimensional space-time obtained from the compactification of type IIA superstring on the K3 surface. In this compactification, we can produce such membranes by considering a D-brane system consisting of:

- Unwrapped D2-branes

- D4-branes wrapping 2-spheres inside the K3 surface

- D6-branes wrapping the K3 surface.

After deleting the zeros of the Hodge diamond of the K3 surface, these 2-branes can be repre- 
sented by the following Hodge brane configurations 1

$D 6$

In what follows, we shall show that the compactification of type IIA superstring on the K3 surface provides a natural realization of the QHE in $1+2$ dimensions. From (3.1), we can engineer at least two type IIA stringy realizations of this effect. The vertical line of the previous Hodge sub-diamond represents a brane system involving D2 and D6-branes wrapping the K3 surface, while the horizontal one considers only D4-branes wrapping intersecting 2spheres. Here we give the vertical representation and leave the horizontal one for the next section. Roughly, the vertical QHE stringy realization can be done in a similar manner as the uncompactified stringy one discussed previously. To see that consider an unwrapped D2-brane which can be obtained from the ten dimensional one. As in the uncompactified case, its worldvolume will play the role of the 3-dimensional space-time on which QHE may reside. However, the magnetic source in six dimensions should have only two extended directions. This would be obtained by compactifying the ten dimensional brane objects on cycles of the K3 surface. The presence of the membrane magnetic source is due to the fact that the electric/magnetic duality in six dimensions reads as

$$
p+q=2 .
$$

In the compactification on the K3 surface, the magnetic membranes, dual to electrical particles in six dimensions, are obtained by two different ways. They are formed by wrapping D4-branes on the 2-spheres of the K3 surface, or by wrapping D6-branes on the entire K3 surface, and they lead to a source of the dual 4-form field strength. These brane objects carry magnetic charges of the $U(1)$ gauge symmetry obtained from the reduction of the corresponding higher dimensional R-R gauge fields.

In this section we restrict our-self to the case of the wrapped D6-branes to form a magnetic source and leave the D4-brane case for the next section. Indeed, consider $N$ coincident of D6-branes wrapping the K3 surface. Wrapping a D6-brane on the K3 surface means that four spatial directions are wrapped on the K3 surface, while the remaining two directions stay unwrapped in six dimensions. As in ten dimensions, we need to turn on fundamental strings connecting these wrapped D6-branes with the D2-brane filling the 3-dimensional space-time on

\footnotetext{
${ }^{1}$ Note in passing that these brane configurations has been used in the study of attractor horizon geometries of extremal black 2-branes [12. In such limits, type IIA backgrounds can be identified with the factorization $A d S_{4} \times S^{2} \times K 3$.
} 
which the QHE lives. The end of the fundamental strings stretched on the D2-brane can be considered as 3-dimensional electrons. We expect to have a realization similar to the one given in ten dimensions. This can be obtained by replacing the unwrapped D6-branes by wrapped D6branes on the K3 surface. Since the K3 surface is not flat, there are some curvature corrections to the brane dynamics which should be taken into account. In fact, it has been shown in [19] that the wrapping of a D6-brane on the K3 surface induces a D2-brane with negative charge within the uncompactified part. The later is not an anti D2-brane since it preserves the same supersymmetries that a D2-brane with that orientation. However, we can add extra D2-branes to the unwrapped part of the D6-branes with the charge number $M$. In this way, the net D2brane charge as measured at infinity is therefore $M-K$. Assuming that this charge number is positive, the filling fraction can be given by the following ratio

$$
\nu=\frac{M-K}{N} \text {. }
$$

If we take $K=N$, this can be reduced to

$$
\nu=k-1, \quad k=\frac{M}{N} .
$$

In order to be able to reach the plateau in which the conductivity is quantized we need a strong enough magnetic field. In our stringy construction this means that the physical distance between the D2-branes and the D6 branes must be small. The fundamental string is streched having one end on the D6 stack and the other end on the D2 stack. If the distance between both stacks is small, the length of the fundamental string is correspondingly small and has quantum vibrations. On the other hand if the distance between both stacks is large (compared with the string length) the string is strongly strched and develops a classical configuration.

Parity is broken on the $2+1$ dimensional space-time developed on the D2 branes by the presence of the strong magnetic field orthogonal to the space. This field is sourced by the magnetic D6-branes. As in [11, another way to break parity is to add a new stack of D4branes wrapping two-cycles on the K3 surface.

Finally, the model presented in this section could be related to other brane realization given recently in [11] based on D3/D7 systems and using the holographic principle. We expect this can be done by T-dualizing along the direction in which the fundamental string lives. In fact if we consider this direction as a compact one, this duality exchanges the type IIA superstring with the type IIB. Moreover, the spacial dimension of the D-brane should change by an odd number because under T-duality the Dirichlet and Neumann boundary conditions are exchanged. After a T-duality on a circle, which can be identified with the fundamental string direction, the D2/D6 system can be converted into the D3/D7 one. On the other hand, string-string duality relating type IIA superstring compactified on the K3 surface with the heterotic superstring compactified on $T^{4}$ should also give dual versions of the model presented here. We believe that these facts deserves to be studied further. 


\section{Hierarchical Type IIA Stringy Description of the QHE}

So far, we have discussed a QHE description with a single gauge group. Here, we give stringy realizations involving multiple $U(1)$ gauge symmetry. This is known by the hierarchical description [20]. Apriori there are many ways to embed QHE abelain gauge theory with $U(1)^{n}$ gauge group in type IIA superstring compactification. A way, which has been discussed in [11, consists of getting abelian gauge fields from the reduction of the $\mathbf{R}-\mathbf{R} 3$-form on 2-spheres of the K3 surface. However, the method presented here is somewhat different. It is based on the study of quiver gauge theories living on the world-volume of the wrapped D4-branes. To do so, consider a local IIA geometry where the K3 surface develops singularities classified by Kac-Moody Lie algebras. In this limit, the K3 surface can be replaced by ALE spaces with $A D E$ singularities. The deformation of such geometries consists on blowing up the singularity in question by a collection of intersecting 2-spheres according to the $A D E$ Dynkin diagrams. In this way, the root system $\left\{\alpha_{i}\right\}$ of the $A D E$ Lie algebras can play a crucial role in the understanding of the K3 algebraic geometry and the corresponding quiver gauge theories embedded in type IIA superstring compactification. It follows that, there is a one to one correspondence between the $A D E$ Lie algebras and the $A D E$ geometries of local limits of the K3 surface. More precisely, to each root $\alpha_{i}$ (say the $s u(n)$ simple root $\alpha_{i}=e_{i}-e_{i+1}$ ) corresponds a 2-sphere $S_{i}^{2}$ of the $A D E$ geometries of the ALE spaces. The connection is supported by the following 2-sphere intersection relation

$$
S_{i}^{2} \cdot S_{j}^{2}=-\alpha_{i} \cdot \alpha_{j}=-K_{i j}
$$

where $K_{i j}$ is exactly the Cartan matrix of the $A D E$ Lie algebras.

Roughly, the abelian $U(1)^{n}$ gauge symmetry in three dimensions, that we consider here, can be obtained by taking a low energy limit of wrapped D4-branes on intersecting geometries. To get that, consider $N$ D4-branes embedded in type IIA superstring. On theirs unwrapped world-volume lives a 5-dimensional gauge theory with $U(N)$ symmetry. In type IIA superstring moving on the deformed $A D E$ backgrounds, the D4-branes can be partitioned into $N_{i}$ D4branes wrapping intersecting 2-spheres arranged as Dynkin diagrams. These wrapped branes fill the 3-dimensional space-time on which type IIA QHE can reside. In this way, the $U(N)$ gauge group breaks down to $\prod_{i=1}^{n} U\left(N_{i}\right)$ where $n$ is the number of the intersecting 2-spheres. Now, identifying $n$ with the number of D4-branes, the gauge group living on the unwrapped part of the world-volume of D4-branes can be totally broken to $U(1)^{n}$ gauge symmetry. This can be obtained by thinking that each single D4-brane wraps only one 2-sphere inside the K3 surface.

As in [11, to couple the system to an external gauge field, we should add an extra D4-brane wrapping a particular 2-cycle playing the role of a magnetic source. As before, this wrapped D4-brane is required by the fact that the total space-time of uncompactified string theory is 
six. Its unwrapped directions should be placed perpendicularly to the uncompactified part of the word-volume of D4-branes on which the QHE resides. As in the previous realization, the missing spatial dimension can be identified with the fundamental string connecting this new wrapped D4-brane with the ones on which the $U(1)^{n}$ gauge theory lives. The full brane system can be described by the $U(1)^{n}$ Chern Simon gauge theory with the following action

$$
S=\frac{1}{4 \pi} \int \sum_{i, j} K_{i j} A^{i} \wedge d A^{j}+\sum_{i} q_{i} \tilde{A} \wedge d A^{i} .
$$

In this action, $\tilde{A}$ is an external $U(1)$ gauge field and $A^{i}$ are dynamical gauge fields. $K_{i j}$ is a $n \times n$ matrix which can be related to the intersection matrix of 2 -spheres within the K3 surface [11]. $q_{i}$ is the electromagnetic charge carried by the extra D4-brane wrapping a special 2-cycle described by a linear combination of $S_{i}^{2}$

$$
\left[C_{2}\right]=\sum_{i} q_{i}\left[S_{i}^{2}\right]
$$

where $S_{i}^{2}$ denote a basis of $H_{2}(K 3, Z)$. Following Wen-Zee mode [21], $K_{i j}$ and $q_{i}$ are interpreted as order parameters classifying the various $\mathrm{FQH}$ states and are related to the filling fraction by

$$
\nu=q_{i} K_{i j}^{-1} q_{j}
$$

From this quantity, we clearly see that the filling fraction is related to the geometric data of type IIA local backgrounds through the intersection numbers of the 2-spheres. Up to some details, these intersection numbers are classified by the Cartan matrices. Following [14], the possible forms of $K_{i j}$ may be grouped basically into three categories. In the language of the Kac-Moody algebras, these correspond to:

1. Cartan matrices of the finite dimensional Lie algebras satisfying det $K>0$

2. Cartan matrices for the affine Lie algebras with the condition $\operatorname{det} K=0$

3. Cartan matrices for the indefinite Lie algebras where det $K<0$.

In terms of Dynkin diagrams of the trivalent $T_{p, q, r}$ extended Lie algebras, det $K$ can have a nice expression. Note that the Dynkin diagram of the $T_{p, q, r}$ Lie algebras involves three ordinary $A_{p}$, $A_{q}$ and $A_{r}$ Dynkin chains glued at a trivalent vertex

The determinant of the Cartan matrix of the corresponding Lie algebra takes the form

$$
\operatorname{det} K\left(T_{p, q, r}\right)=p q r \Gamma
$$

where $\Gamma$ is given by the following quantity

$$
\Gamma=\frac{1}{p}+\frac{1}{q}+\frac{1}{r}-1 .
$$




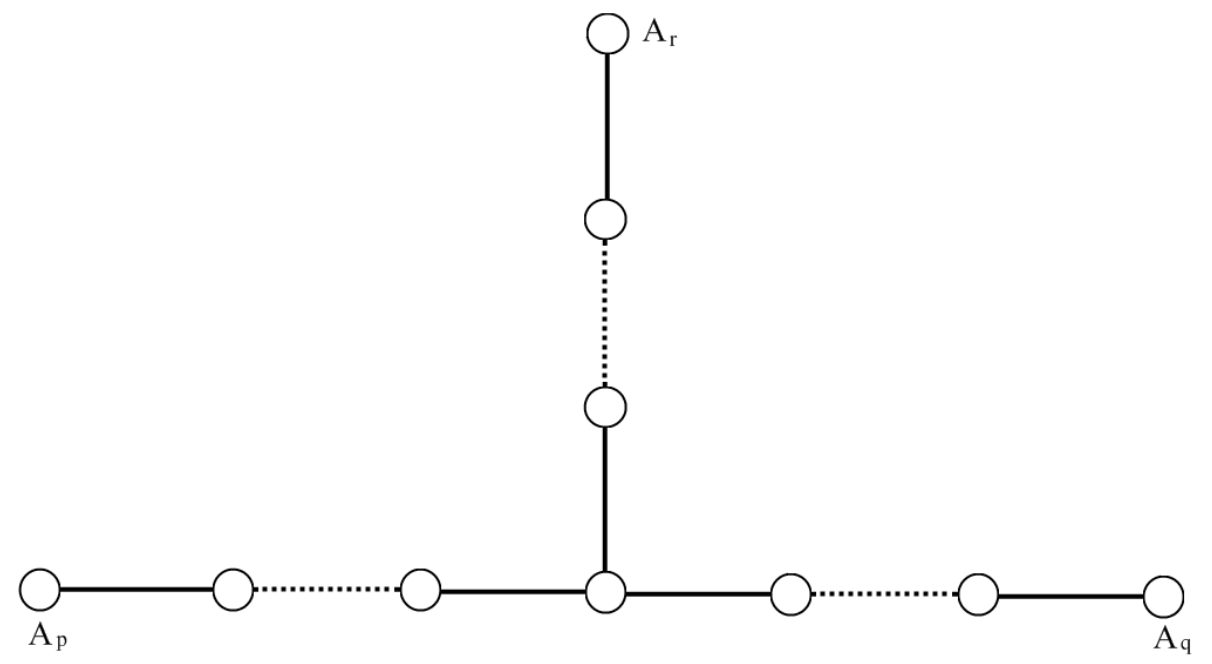

In this way, the above classification can be reformulated as follows:

1. Cartan matrices of the finite dimensional Kac-Moody Lie algebras satisfying $\Gamma>0$

2. Cartan matrices for the affine Kac-Moody Lie algebras are given by the condition $\Gamma=0$

3. Cartan matrices for indefinite Kac-Moody Lie algebras are defined by $\Gamma<0$.

From both classifications, it follows that no QHE in $1+2$ dimensions can occur in the quiver gauge theory of D4-branes wrapping on intersecting 2-spheres according to the affine Dynkin diagrams.

However, the finite and the indefinite ones give some QHE stringy realizations. In particular, one may distinguish between two models depending on the values of the filling fraction. The first model involves a quiver theory encoded in Dynkin diagrams of finite Lie algebras. This leads to a fractional QHE with positive values. It is best to give an example. Consider a particular vector charge like $q_{i}=(1, \ldots, 0)$. In this way, the above system reduces to a model with the filling fraction given by

$$
\nu(A D E)=K_{11}^{-1} .
$$

In the case of the finite Lie algebra $A_{n}$, the corresponding wrapped geometry consists of $n$ intersecting 2-spheres as

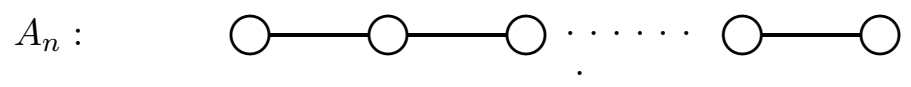

This admits a $T_{n, 1,1}$ realization and its Cartan matrix reads $K_{i j}$ reads as

$$
K_{i j}=-\delta_{i-1, j}+2 \delta_{i, j}-\delta_{i+1, j}
$$

Using equations (4.5) and (4.6), its determinant is given by

$$
\operatorname{det} K\left(T_{n, 1,1}\right)=n+1 \text {. }
$$


From the matrix inverse, it is easy to see that the corresponding filling fraction reduces to

$$
\nu\left(A_{n}\right)=\frac{n}{n+1} .
$$

It should be interesting to note that one can get filling fractions with odd-denominator using a special choice of the singularities of ALE spaces. In the case of the finite $A_{n}$ geometries, we recover some very known values observed experimentally including the Jain series given by

$$
\nu^{\text {Jain }}=\frac{m}{2 p m \pm 1}
$$

Specializing the computation to the same vector charge as before and taking $n=2 m$, we obtain $\nu=\frac{2 m}{2 m+1}$ which coincides with two times the most stable part of the Jain's sequence with $p=1$. The full series can be recovered exactly if we replace the matrix $K_{i j}$ that appears in the action (4.2) by $2 K_{i j}$. In the algebraic side, this can be obtained by fixing the normalization of the inner product to be such that the highest weight of the adjoint representation has norm 1. It is worth noting that other series can be obtained in the context of M-theory compactified on eight dimensional manifolds. This will be given in future works.

The second model is described by a quiver gauge theory based on indefinite geometries and gives QHE stringy realization with negative filling factor. This negative value could have a physical interpretation in the physics of semi-conductors. To see that consider a simple example of the indefinite Lie algebras given by the hyperbolic symmetry $\left(\widehat{H A}_{2}\right)$. The derivation of this hyperbolic geometry is based on the same philosophy one uses in the building of the affine Dynkin diagrams from the finite ones by adding a node. In other words, by cutting this node in $\widehat{H A}_{2}$, the resulting sub-diagram coincides with the affine Lie algebra $\widehat{A}_{2}$. The new Dynkin graph looks like

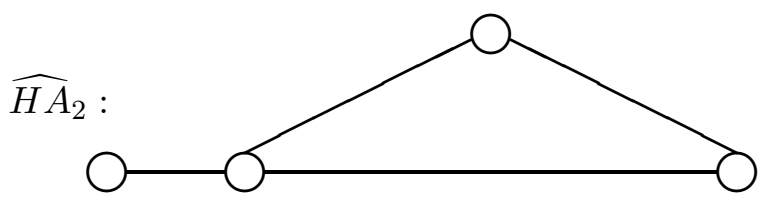

Its Cartan matrix takes the form

$$
K_{i j}\left(\widehat{H A}_{2}\right)=\left(\begin{array}{cccc}
2 & -1 & 0 & 0 \\
-1 & 2 & -1 & -1 \\
0 & -1 & 2 & -1 \\
0 & -1 & -1 & 2
\end{array}\right)
$$


and its inverse is given by

$$
K_{i j}^{-1}\left(\widehat{H A}_{2}\right)=\left(\begin{array}{cccc}
0 & -1 & -1 & -1 \\
-1 & -2 & -2 & -2 \\
-1 & -2 & -\frac{4}{3} & -\frac{5}{3} \\
-1 & -2 & -\frac{5}{3} & -\frac{4}{3}
\end{array}\right) .
$$

Now, using equation (4.4) and taking a vector charge like $q_{i}=(0,1,0,0)$, the corresponding filling fraction is

$$
\nu\left(\widehat{H A}_{2}\right)=-2
$$

This value could be related to some results in the physics of graphene. The latter is a monoatomic layer of graphite with Carbon atoms arranged in tow dimensional honeycomb lattice representation. The electronic structure of the graphene can be modelled by fermions localized in two dimensions. We expect that the above negative value $\nu=-2$ can be related to holes in the graphene studied in [22]. The model with $\nu=2$ might be related to it by particle-hole symmetry. It would therefore be of interest to try to extract more information on this string theory conection with graphene. We believe that this conection deserves to be studied further.

\section{Discussions and open questions}

In this paper, we have engineered stringy realizations of the QHE in $1+2$ dimensions from the compactification of type IIA superstring on the K3 surface. Using the results of the attractor horizon geometries of the extremal black 2-branes on the Calabi-Yau manifolds and based on the Hodge diamond of the K3 surface, we have given two different realizations of this effect. In particular, we have presented hierarchical stringy descriptions using quiver gauge models living on a stack of D4-branes wrapping intersecting 2-spheres which are classified by Cartan matrices of Lie algebras. These representations could be related to the realization of the QHE in $1+5$ dimensions involving unwrapped D4-branes in ten dimensions [6]. This may be done by wrapping D4-branes on the 2-spheres in the compactification on the K3 surface.

Using ideas borrowed from the classification of K3 surface singularities, we have studied the QHE realizations with filling fractions depending on local type IIA intersecting geometries. As results, we have found that there are no QHE stringy descriptions based on type IIA geometries corresponding singularities classified by affine Kac-Moody Lie algebras. The stringy realizations of QHE can be done only for the finite and indefinite geometries. In particular, the finite Lie algebras are associated with fractional filling factors; while the indefinite ones classify models with negative filling factors, which could be related to the physics of holes in the graphene. It should be interesting to develop this observation. This can go in the same sense of the string 
theory realization of the graphene using of D3-D7 brane system studied recently in [23]. It would also be interesting to understand the link between our results and the ones in [24] based on dyonic black holes.

An interesting question for further investigations concerns a direct derivation of the QHE in $1+2$ from string theory compactified on seven real dimensional manifolds. To make contact with this present work, a possible candidate could be G2 manifolds with K3-fibrations. On the other hand, the result of this paper could be up lifted to M-theory. In this way, the QHE configurations can be reproduced by wrapping M2 and M5-branes on appropriate cycles in the K3 surface. It would be also worth investigating a direct compactification on eight dimensional manifolds. A special interest might be devoted to incorporate the compactification on CalabiYau fourfolds or Spin(7) manifolds with K3-frabations.

On the other hand, motivated by results of supersymmetric matrix model of the QHE given in [25], it should be interesting to study realizations in terms of local K3 supersurface with $A D E$ geometries studied in [26]. We hope, all these open questions will be addressed in future work.

Acknowledgments. This work has been supported CICYT (grant FPA2009-09638), DGIID-DGA (grant 2007-E24/2) and PCI-AECI (grant 07 and 10). We thank M. Asorey, L. J. Boya, I. Cavero-Peláez, M. P. Garcia del Moral, J. M. Munoz-Castaneda, E. H. Saidi, L. Segui for discussions and collaborations. AB would like to thank S. J. Rey for sending him the paper [23]. He would also thank Montanez-Naz family for very kind hospitality.

\section{References}

[1] L. Susskind, The Quantum Hall fluid and Noncommutative Chern-Simons Theory, hep-th/0101029.

[2] B.A. Bernevig, J. Brodie, L. Susskind and N. Toumbas, How Bob Laughlin Tamed the Giant Graviton from Taub-NUT space, JHEP0102(2001)003, hep-th/0010105.

[3] I. Bena, A. Nudelman, On the stability of the Quantum Hall soliton, JHEP 0012(2000)017, hep-th/0011155.

[4] S. Hellerman and L. Susskind, Realizing the quantum Hall system in string theory, hep-th/0107200.

[5] O. Bergman, Y. Okawa and J. H. Brodie, The stringy quantum Hall fluid, JHEP 0111 (2001) 019, hep-th/0107178. 
[6] M. Fabinger, Higher-Dimensional Quantum Hall Effect in String Theory, JHEP 0205 (2002) 037, hep-th/0201016.

[7] O. Bergman, Quantum Hall Physics in String Theory, hep-th/0401106.

[8] J. L. Davis, P. Kraus and A. Shah, Gravity Dual of a Quantum Hall Plateau Transition, JHEP 0811 (2008) 020, arXiv:0809.1876 [hep-th].

[9] S. A. Hartnoll and P. Kovtun, Hall conductivity from dyonic black holes, Phys. Rev. D76 (2007) 066001, arXiv:0704.1160 [hep-th].

[10] R. Abounasr, M. Ait Ben Haddou, A. El Rhalami, E. H. Saidi, Algebraic Geometry Realization of Quantum Hall Soliton, J.Math.Phys. 46(2005)022302, hep-th/0406036.

[11] M. Fujita, W. Li, S. Ryu, T. Takayanagi, Fractional Quantum Hall Effect via Holography: Chern-Simons, Edge States, and Hierarchy, arXiv:0901.0924[hep-th].

[12] A. Belhaj, L. B. Drissi, E. H. Saidi, A. Segui, N=2 Supersymmetric Black Attractors in Six and Seven Dimensions, Nucl. Phys. B796(2008)521-580, arXiv:0709.0398[hep-th].

[13] R. Ahl Laamara, M. Asorey, A. Belhaj, A. Segui, Extremal Black Brane Attractors on The Elliptic Curve, J. Phys. A: Math. Theor. 43 (2010)00), arXiv:0907.0093[hep-th].

[14] V. G. Kac, Infinite dimensional Lie algebras, third edition, Cambridge University Press (1990).

[15] M. Ait Ben Haddou, A. Belhaj, E.H. Saidi, Classification of $N=2$ supersymmetric CFT(4)s: Indefinite series, J. Phys. A38(2005)1793-1806, hep-th/0308005.

[16] R. Ahl Laamara, M. Ait Ben Haddou, A Belhaj, L.B Drissi, E.H Saidi, RG Cascades in Hyperbolic Quiver Gauge Theories, Nucl. Phys. B702 (2004)163-188, hep-th/0405222.

[17] A. Hanany and E. Witten, Nucl. Phys. B492 (1997) 152, hep-th/ 9611230.

[18] P. S. Aspinwall, K3 Surfaces and String Duality, hep-th/9611137.

[19] C. V. Johnson, A. W. Peet and J. Polchinski, Gauge theory and the excision of repulson singularities, Phys. Rev. D 61 (2000) 086001, hep-th/9911161.

[20] B. Halprin, Statistics of Quasiparticles and the hierarchy of fractional Quan- tum Hall States, Phys. Rev. Lett. 52(1984)1583-1586.

[21] X.G. Wen, A. Zee, Classification of Abelian quantum Hall states and matrix formulation of topological fluids, Phys. Rev. B 46(1992)2290-2301. 
[22] V. Singh, M. M. Deshmukh, Non-equilibrium breakdown of quantum Hall state in graphene, Phys. Rev. B 80(2009) 081404, arXiv:0908.0074[cond-mat].

[23] S.J. Rey, String Theory on Thin Semiconductors: Holographic Realization of Fermi Points and Surfaces, Prog. of Theo. Phy. [Supp.] 177(2009)128-142, arXiv:0911.5295[hep-th].

[24] D. Bak, S. J. Rey, Composite Fermion Metals from Dyon Black Holes and S-Duality, arXiv:arXiv:0912.0939

[25] J. Gates Jr, A. Jellal, E. H. Saidi, M. Schreiber, Supersymmetric Embedding of the Quantum Hall Matrix Model, JHEP 0411 (2004) 075, hep-th/0410070.

[26] R. Ahl Laamara, A. Belhaj, L.B. Drissi, E.H. Saidi, On Local Calabi-Yau Supermanifolds and Their Mirrors, J. Phys. A39(2006) 5965-5978, hep-th/0601215. 